\title{
Fisiopatologia da trombose associada à infecção pelo SARS-CoV-2
}

\author{
Physiopathology of SARS-CoV-2-infection-associated thrombosis \\ Ivan Benaduce Casella ${ }^{1}$
}

\begin{abstract}
Como citar: Casella IB. Fisiopatologia da trombose associada à infecção pelo SARS-CoV-2.J Vasc Bras. 2020;19:e20200128. https://doi.org/10.1590/1677-5449.200128
\end{abstract}

A pandemia provocada pelo novo coronavírus (SARS-CoV-2) é o evento mais severo de saúde pública das últimas décadas, com mais de 11,1 milhões de casos contabilizados em todo o mundo até o início de julho de 2020 e mais de 528 mil mortes $^{1}$. As manifestações clínicas da infecção pelo SARS-CoV-2 são múltiplas, assim como os processos fisiopatológicos que as proporcionam. Desde o início, o número de eventos trombóticos de diversas naturezas tem chamado a atenção de médicos e pesquisadores, evocando a necessidade de sua compreensão para que os desfechos clínicos mais sombrios sejam evitados.

Este editorial tem a modesta pretensão de apresentar as teorias e evidências científicas acerca da fisiopatologia dos processos trombóticos associados à infecção pelo SARS-CoV-2. No entanto, há alguns aspectos importantes a serem realçados: parte das explicações cientificas ainda se encontram no campo das hipóteses; os processos patológicos observados são predominantemente concomitantes e se estimulam mutuamente; ainda é difícil quantificar o impacto isolado de eventos que ocorrem em escala molecular e celular nos desfechos clínicos finais do evento trombótico.

\section{PAPEL DA ENZIMA DE CONVERSÃO DA ANGIOTENSINA TIPO 2 (ECA2)}

O principal mecanismo de acesso do SARS-CoV-2 ao meio intracelular se dá pela interação de sua glicoproteína de superfície $\mathrm{S}$ com a glicoproteína humana ECA2, presente tanto no plasma como na membrana de diversos tipos celulares ${ }^{2}$. Nesse processo, a ECA2 atua como receptor de membrana, contando ainda com a interação de outros efetores, como a serina protease transmembrana tipo $2^{3}$.

A proteína ECA2 cumpre importante papel de efetor negativo no sistema renina angiotensina aldosterona, convertendo as angiotensinas I e II em angiotensinas 1-9 e 1-7, respectivamente. As angiotensinas 1-9 e 1-7 apresentam efeitos vasodilatadores e antiinflamatórios, dentre outros, antagonizando, assim, os efeitos clássicos hipertensivos e inflamatórios da angiotensina $\mathrm{II}^{2,3}$.

A infecção pelo SARS-CoV-2 resulta em morte de células ricas em receptores ECA2 e na internalização celular de parte desses receptores, causando, em última instância, redução da atividade da ECA2 circulante ${ }^{4}$. Com isso, há um predomínio da atividade da angiotensina II sobre as angiotensinas 1-7 e 1-9. A angiotensina II, além de seu efeito hipertensor e inflamatório, estimula a ativação da cascata da coagulação pela via do fator tissular (FT), eleva a atividade do inibidor do ativador do plasminogênio tecidual tipo 1 (PAI-1) e inibe a expressão do ativador do plasminogênio tecidual (tPA $)^{5}$.

\section{IMUNOTROMBOSE E REDES EXTRACELULARES DE NEUTRÓFILOS}

O termo "imunotrombose" tem sido utilizado para descrever a interação entre macrófagos, polimorfonucleares, plaquetas, fatores de coagulação e proteínas imunoefetoras, formando trombos na microvasculatura com o intuito de identificar patógenos e restringir mecanicamente a sua propagação ${ }^{6}$. A imunotrombose é usualmente desencadeada por agentes infecciosos circulantes no sangue, podendo também ser acionada em infecções virais.

Os polimorfonucleares envolvidos nesse processo estimulam a formação de redes extracelulares de neutrófilos, que podem estimular a ativação da coagulação via fator XII atuando também na inibição de proteínas anticoagulantes endógenas ${ }^{5}$. Zuo et al. observaram que elevados níveis de ativação de neutrófilos e formação de redes extracelulares de neutrófilos em pacientes 
positivos para Covid (Covid+) foram associados a maior risco de complicações trombóticas ${ }^{7}$.

\section{RESPOSTA ENDOTELIAL, INFLAMAÇÃO E TROMBOSE}

Ackermann et al. ${ }^{8}$ demonstraram que infecção pulmonar pelo SARS-CoV-2 está associada a microtrombose, endotelite e angiogênese intussusceptiva, em uma intensidade não observada em outras infecções virais ou pneumonias intersticiais de semelhante gravidade. $\mathrm{O}$ endotélio vascular em condições fisiológicas dispõe de diversos mecanismos inibidores de eventos trombóticos. De forma oposta, estímulos patológicos em nível celular ou molecular estimulam uma resposta trombogênica endotelial, como o aumento da expressão do FT e do PAI-1, ativação plaquetária, liberação de citocinas inflamatórias e redução de expressão de anticoagulantes endógenos, como a trombomodulina 9 .

Essas citocinas inflamatórias também são maciçamente liberadas por macrófagos alveolares, células epiteliais e polimorfonucleares ${ }^{10}$ pela resposta imunológica tardia e exacerbada mediada por interferons ${ }^{11}$. Realimentando o círculo vicioso da inflamação e trombose, as citocinas são fatores quimiotáticos para leucócitos, ativam a coagulação pela via do fator tissular, inibem a fibrinólise ao elevar a expressão do PAI-1 e inibem vias anticoagulantes endógenas, como a antitrombina, a proteína $\mathrm{C}$ e seu cofator, a proteína $\mathrm{S}^{10,12}$.

O sistema complemento é um dos elementos da resposta imune na infecção pelo SARS-CoV-2 e também está associado aos processos trombóticos observados. Magro et al.$^{13}$ observaram intensa atividade do sistema complemento em pacientes críticos positivos para SARS-CoV-2, com deposição de frações C5b-9 e $\mathrm{C} 4 \mathrm{~d}$ na microvasculatura, associadas à microtrombose com deposição de fibrina e lesão endotelial.

\section{DÍMERO D}

A marcante elevação do dímero $\mathrm{D}$ foi observada em pacientes Covid + , sendo superlativa nos pacientes mais graves e claramente associada a piores prognósticos ${ }^{14}$. Embora não totalmente compreendida, pode ser explicada pela ocorrência de maciça trombose microvascular e pela ativação do sistema fibrinolítico por estímulo viral direto com posterior inibição deste ${ }^{14,15}$.

Resumidamente, podemos agrupar os efeitos dos eventos descritos em quatro resultantes principais agindo sobre a coagulação: (1) ativação da cascata da coagulação por vias distintas e múltiplos estímulos de origens distintas; (2) ativação plaquetária; (3) inibição das proteínas anticoagulantes endógenas (proteína $\mathrm{C}$ e seu cofator proteína $\mathrm{S}$, antitrombina, inibidor da via do fator tissular); (4) inibição do sistema fibrinolítico (“fibrinolysis shutdown").

\section{INFECÇÃO PELO SARS-COV-2, EVENTOS TROMBÓTICOS E RESISTÊNCIA À HEPARINA}

Diversos autores ${ }^{16-18}$ reportaram aumento de eventos trombóticos tanto venosos (trombose venosa profunda, embolia pulmonar) quanto arteriais (acidente vascular encefálico isquêmico, tromboses arteriais periféricas) em pacientes infectados pelo SARSCoV-2. Tais relatos diferem bastante em seus métodos, o que impede a obtenção de dados homogêneos. Helms et al. ${ }^{19}$ observaram incidência aumentada de eventos de tromboembolismo venoso em pacientes graves infectados pelo SARS-CoV-2 em comparação com pacientes com infecções respiratórias severas causadas por outros patógenos.

White et al. ${ }^{20}$ observaram um pequeno coorte de pacientes infectados pelo SARS-CoV-2 recebendo anticoagulação terapêutica com heparinas. Neste grupo, 5 em 5 pacientes tratados com enoxaparina apresentaram níveis de atividade antifator Xa inferiores aos esperados para ação terapêutica da droga. Para a heparina não fracionada, $8 \mathrm{em} 10$ pacientes apresentaram resistência à anticoagulação.

De maneira similar, Dutt et al. ${ }^{21}$ notaram que $27 \%$ dos pacientes com infecção não severa pelo SARSCoV-2 recebendo doses profiláticas de heparina apresentavam valores de atividade antifator $\mathrm{Xa}$ abaixo dos níveis indicadores de efetividade da profilaxia. Já nos pacientes mais graves, internados em ambiente de terapia intensiva, a porcentagem era de $95 \%$.

\section{CONCLUSÕES}

As evidências atuais indicam que existe uma expressiva incidência de eventos trombóticos clinicamente relevantes associados à infecção pelo SARS-CoV-2. A compreensão dos processos fisiopatológicos que resultam em tais fenômenos trombóticos ainda é incompleta. Também, a relação de causa e efeito entre eventos trombóticos e a gravidade da infecção pelo SARS-CoV-2 ainda precisa ser mais bem compreendida. Não está claro se são os pacientes com maior resposta trombótica que apresentam os quadros clínicos mais severos ou se o processo é sequencialmente oposto, em que os casos clinicamente mais graves resultam em maior incidência de eventos trombóticos.

\section{REFERÊNCIAS}

1. World Health Organization. Coronavirus disease (COVID-19): Situation Report - 167. Geneva: WHO; 2020 [cited 2020 July 20]. 
https://www.who.int/docs/default-source/coronaviruse/situationreports/20200705-covid-19-sitrep-167.pdf?sfvrsn=17e7e3df_4

2. Hoffmann $M$, Kleine-Weber $\mathrm{H}$, Schroeder $\mathrm{S}$, et al. SARS-CoV-2 cell entry depends on ACE2 and TMPRSS2 and is blocked by a clinically proven protease inhibitor. Cell. 2020;181(2):271-280.e8. http://dx.doi.org/10.1016/j.cell.2020.02.052. PMid:32142651.

3. Xiao L, Sakagami H, Miwa N. ACE2: the key molecule for understanding the pathophysiology of severe and critical conditions of COVID-19: Demon or Angel? Viruses. 2020;12(5):491. http:// dx.doi.org/10.3390/v12050491. PMid:32354022.

4. Gheblawi M, Wang K, Viveiros A, et al. Angiotensin-converting enzyme 2: SARS-CoV-2 receptor and regulator of the reninangiotensin system: celebrating the 20th Anniversary of the Discovery of ACE2. Circ Res. 2020;126(10):1456-74. http://dx.doi. org/10.1161/CIRCRESAHA.120.317015. PMid:32264791.

5. Henry BM, Vikse J, Benoit S, Favaloro EJ, Lippi G. Hyperinflammation and derangement of renin-angiotensin-aldosterone system in COVID-19: a novel hypothesis for clinically suspected hypercoagulopathy and microvascular immunothrombosis. Clin Chim Acta. 2020;507:167. 73. http://dx.doi.org/10.1016/j.cca.2020.04.027. PMid:32348783.

6. Engelmann B, Massberg $S$. Thrombosis as an intravascular effector of innate immunity. Nat Rev Immunol. 2013;13(1):34-45. http:// dx.doi.org/10.1038/nri3345. PMid:23222502.

7. Zuo Y, Zuo M, Yalavarthi S, et al. Neutrophil extracellular traps and thrombosis in COVID-19. medRxiv. 2020 May 5. Epub ahead of print. http://dx.doi.org/10.1101/2020.04.30.20086736. PMid:32511553.

8. Ackermann $M$, Verleden $S E$, Kuehnel $M$, et al. Pulmonary vascular endothelialitis, thrombosis, and angiogenesis in Covid-19. N Engl J Med. 2020;383(2):120-8. http://dx.doi.org/10.1056/NEJMoa2015432. PMid:32437596.

9. Jackson SP, Darbousset R, Schoenwaelder SM. Thromboinflammation challenges of therapeutically targeting coagulation and other host defense mechanisms. Blood. 2019;133(9):906-18. http://dx.doi. org/10.1182/blood-2018-11-882993. PMid:30642917.

10. Tay MZ, Poh CM, Rénia L, MacAry PA, Ng LFP. The trinity of COVID-19: immunity, inflammation and intervention. Nat Rev Immunol. 2020;20(6):363-74. http://dx.doi.org/10.1038/s41577020-0311-8. PMid:32346093.

11. McGonagle D, Sharif K, O'Regan A, Bridgewood C. The Role of Cytokines including Interleukin-6 in COVID-19 induced Pneumonia and Macrophage Activation Syndrome-Like Disease. Autoimmun Rev. 2020;19(6):102537. http://dx.doi.org/10.1016/j. autrev.2020.102537. PMid:32251717.

12. Levi $M$, van der Poll $T$, ten Cate $H$, van Deventer SJ. The cytokinemediated imbalance between coagulant and anticoagulant mechanisms in sepsis and endotoxaemia. Eur J Clin Invest. 1997;27(1):3-9. http://dx.doi.org/10.1046/j.1365-2362.1997.570614.x. PMid:9041370.
13. Magro C, Mulvey JJ, Berlin D, et al. Complement associated microvascular injury and thrombosis in the pathogenesis of severe COVID-19 infection: a report of five cases. Transl Res. 2020;220:113. http://dx.doi.org/10.1016/j.trsl.2020.04.007. PMid:32299776.

14. Tang N, Li D, Wang X, Sun Z. Abnormal coagulation parameters are associated with poor prognosis in patients with novel coronavirus pneumonia. J Thromb Haemost. 2020;18(4):844-7. http://dx.doi. org/10.1111/jth.14768. PMid:32073213.

15. Wright FL, Vogler TO, Moore EE, et al. Fibrinolysis shutdown correlation with thromboembolic events in severe COVID-19 infection. J Am Coll Surg. 2020:231(2):203-4. https://doi.org/10.1016/j. jamcollsurg.2020.05.007.

16. Klok FA, Kruip MJHA, van der Meer NJM, et al. Confirmation of the high cumulative incidence of thrombotic complications in critically ill ICU patients with COVID-19: an updated analysis. Thromb Res. 2020;191:148-50. http://dx.doi.org/10.1016/j.thromres.2020.04.041. PMid:32381264.

17. Middeldorp S, Coppens $M$, van Haaps TF, et al. Incidence of venous thromboembolism in hospitalized patients with COVID-19. J Thromb Haemost. 2020;18(8):1995-2002. http://dx.doi.org/10.1111/ jth.14888. PMid:32369666.

18. Zhang L, Feng X, Zhang D, et al. Deep vein thrombosis in hospitalized patients with coronavirus disease 2019 (COVID-19) in Wuhan, China: prevalence, risk factors, and outcome. Circulation. 2020:142(2):11428. https://doi.org/10.1161/CIRCULATIONAHA.120.046702.

19. Helms J, Tacquard C, Severac F, et al. High risk of thrombosis in patients with severe SARS-CoV-2 infection: a multicenter prospective cohort study. Intensive Care Med. 2020;46(6):1089-98. http://dx.doi.org/10.1007/s00134-020-06062-x. PMid:32367170.

20. White D, MacDonald S, Bull T, et al. Heparin resistance in COVID-19 patients in the intensive care unit. J Thromb Thrombolysis. 2020;50(2):287-91. PMid:32445064.

21. Dutt $T$, Simcox D, Downey $C$, et al. Thromboprophylaxis in COVID-19: Anti-FXa - The Missing Factor? Am J Respir Crit Care Med. 2020;202(3):455-7. http://dx.doi.org/10.1164/rccm.2020051654LE. PMid:32510975.

Correspondência Ivan Benaduce Casella Universidade de São Paulo - USP, Instituto Central, Faculdade de Medicina - FM, Hospital das Clínicas - HC, Disciplina de Cirurgia Vascular e Endovascular Rua Ovídio Pires de Campos, s/n, $6^{\circ}$ andar, PAMB, bloco 5, Cerqueira César

CEP 05403-911 - São Paulo (SP), Brasil Tel.: (11) 2661-6101 E-mail: ivan.c@hc.fm.usp.br

Informações sobre o autor IBC - Cirurgião vascular, Hospital das Clínicas, Faculdade de Medicina, Universidade de São Paulo (FMUSP). 